\title{
ENU mutagenesis identifies mice with mitochondrial branched-chain aminotransferase deficiency resembling human maple syrup urine disease

\author{
Jer-Yuarn Wu, ${ }^{1,2}$ Hsiao-Jung Kao, ${ }^{1}$ Sing-Chung Li, ${ }^{1}$ Robert Stevens, ${ }^{3}$ Steven Hillman, ${ }^{3}$ \\ David Millington, ${ }^{3}$ and Yuan-Tsong Chen ${ }^{1,3}$
}

\begin{abstract}
${ }^{1}$ Institute of Biomedical Sciences, Academia Sinica, Taipei, Taiwan, Republic of China ${ }^{2}$ Department of Medical Research, China Medical University Hospital, Taichung, Taiwan, Republic of China ${ }^{3}$ Department of Pediatrics, Duke University Medical Center, Durham, North Carolina, USA
\end{abstract}

\begin{abstract}
Tandem mass spectrometry was applied to detect derangements in the pathways of amino acid and fatty acid metabolism in $N$-ethyl- $N$-nitrosourea-treated (ENU-treated) mice. We identified mice with marked elevation of blood branched-chain amino acids (BCAAs), ketoaciduria, and clinical features resembling human maple syrup urine disease (MSUD), a severe genetic metabolic disorder caused by the deficiency of branched-chain $\alpha$-keto acid dehydrogenase (BCKD) complex. However, the BCKD genes and enzyme activity were normal. Sequencing of branched-chain aminotransferase genes (Bcat) showed no mutation in the cytoplasmic isoform (Bcat-1) but revealed a homozygous splice site mutation in the mitochondrial isoform (Bcat-2). The mutation caused a deletion of exon 2, a marked decrease in Bcat-2 mRNA, and a deficiency in both BCAT-2 protein and its enzyme activity. Affected mice responded to a BCAA-restricted diet with amelioration of the clinical symptoms and normalization of the amino acid pattern. We conclude that BCAT-2 deficiency in the mouse can cause a disease that mimics human MSUD. These mice provide an important animal model for study of BCAA metabolism and its toxicity. Metabolomics-guided screening, coupled with ENU mutagenesis, is a powerful approach in uncovering novel enzyme deficiencies and recognizing important pathways of genetic metabolic disorders.
\end{abstract}

J. Clin. Invest. 113:434-440 (2004). doi:10.1172/JCI200419574.

\section{Introduction}

Although there is much current interest in the genomewide analysis of organisms at the level of transcription (the transcriptome) and translation (the proteome), the third level of analysis, that of the metabolome, has been relatively unexplored to date (1). The term metabolome refers to the entire complement of all the small molecular weight metabolites inside an organism of interest.

Our laboratory has long pioneered the rapid analysis of metabolites at the whole-organism level, using methods such as tandem mass spectrometry, HPLC, and gas chro-

Received for publication July 23, 2003, and accepted in revised form November 11, 2003.

Address correspondence to: Yuan-Tsong Chen, Institute of Biomedical Sciences, Academia Sinica, 128, Academia Road, Section 2, Nankang, Taipei, Taiwan, Republic of China. Phone: 011-886-2-2789-9104; Fax: 011-886-2-2782-5573. E-mail: chen0010@ibms.sinica.edu.tw.

Jer-Yuarn Wu and Hsiao-Jung Kao contributed equally to this work.

Conflict of interest: The authors have declared that no conflict of interest exists.

Nonstandard abbreviations used: $N$-ethyl- $N$-nitrosourea (ENU); branched-chain amino acid (BCAA); maple syrup urine disease (MSUD); branched-chain $\alpha$-keto acid dehydrogenase (BCKD); branched-chain aminotransferase (BCAT); nonfluorescent quencher (NFQ); deoxyribonucleoside triphosphate (dNTP); branched-chain $\alpha$-keto acid (BCKA). matography/mass spectrometry $(2,3)$. Tandem mass spectrometry is a branch of analytic chemistry in which compounds of interest (target analytes) can be detected and analyzed in complex mixture with little or no recourse to on-line chromatography separation. Acylcarnitines reflect the catabolism of fatty acids and certain amino acids, which take place primarily in the mitochondria, the organelle that provides the energy for the cell (2-4). Alpha amino acids share a common "backbone" to which different groups are attached, and they also are amenable to analysis by tandem mass spectrometry (5). Tandem mass spectrometry for amino acids and acylcarnitines determination has been fully automated and can reliably analyze more than 30 metabolites from a single blood spot ( $25 \mu$ l of whole blood) in one shortduration run and thereby provides a comprehensive assessment of the entire fatty acid and selected amino acid metabolic pathways. The method can detect more than 20 metabolic disorders in human $(6,7)$.

A systematic, genome-wide, phenotype-driven mouse mutagenesis program for gene function studies has been described $(8,9)$. Treatment with $N$-ethyl- $N$-nitrosourea (ENU) efficiently generates single-nucleotide mutations in mice, which can be screened for the disease phenotypes of interest. A systematic screening for metabolic diseases with tandem mass spectrometry has been proposed, but no results have been reported thus far (10). 
Here we show how a metabolomics-guided screening of ENU mice can efficiently identify a mouse model of human metabolic disease. We applied the tandem mass spectrometry method to screen thirdgeneration progeny of ENU-mutagenized mice (C57BL/6J) for abnormalities in the pathways of amino acid and fatty acid metabolism.

\section{Methods}

Generation of ENU-recessive mice. ENU-treated mice were bred according to the three-generation breeding scheme as described (11). Briefly, C57BL/6J male mice were given three ENU intraperitoneal injections (100 $\mathrm{mg} / \mathrm{kg}$ body weight) to generate $\mathrm{G} 0$ mice. G0 mice were then mated with normal B6 females to generate G1 male founder mice. Normal B6 females were mated with G1 male founders to generate G2 mice. G2 females were then backcrossed to G1 male mice to generate G3 offspring. Breeding and housing of the mice were conducted in the Mouse Mutagenesis Program Core facility of the Academia Sinica under specific pathogen-free conditions. Animal protocol was approved by the Institutional Animal Safety Committee. Mice were maintained on the regular rodent diet (PicoLab Mouse Diet 20 code5058, PMI LabDiet, St. Louis, Missouri, USA) or branched-chain amino acids-restricted (BCAAs-restricted) diet by mixing regular diet with BCAAs-free human formula (maple syrup urine disease [MSUD] diet powder from Mead Johnson Nutritionals, Evansville, Indiana, USA).

Tandem mass spectrometry screening of amino acids and acylcarnitines. Standardized filter papers (S\&S 903; Schleicher \& Shuell, Dassel, Germany) were impregnated with $25 \mu \mathrm{l}$ whole blood from the tail vein of 2 to 3-month- old G3 mice. Blood was eluted from blood spots, and amino acids and acylcarnitines were derivatized as previously described $(6,7)$. We used a tandem mass spectrometer (Quattro Micro; Micromass, Beverly, Massachusetts, USA) to analyze acylcarnitines and amino acids. Acylcarnitines were detected with a positive precursor ion scan of $85 \mathrm{Da}$, and amino acids were detected by positive neutral loss scans of 102,119 , and $161 \mathrm{Da}$, respectively. The ratios of molecular signals to their respective internal standards were used to quantify the analytes. Amino acids were also determined by ion exchange chromatography on an amino acid analyzer (Beckman Instruments, Palo Alto, California, USA), which enabled the complete separation and quantification of leucine, isoleucine, and allo-isoleucine.

Urinary organic acids. We collected $100 \mu \mathrm{l}$ urine from each mouse. The urine samples were diluted to $1 \mathrm{ml}$, derivatized with ethoxyamine hydrochloride at $\mathrm{pH} 10$, acidified to $\mathrm{pH} 1$, and then extracted four times with $2 \mathrm{ml}$ of ethyl acetate. After evaporation of the combined extracts, the residue was silylated with $100 \mu \mathrm{l}$ of $N, O$-bis(trimethylsilyl) trifluoroacetamide with $1 \%$ trimethylchlorosilane plus $10 \mu \mathrm{l}$ pyridine and analyzed by capillary column gas chro- matography/mass spectrometry, using the analytical conditions described previously (12).

Enzyme assays. Branched-chain $\alpha$-keto acid dehydrogenase (BCKD) activity was assayed spectrophotometrically using $\alpha$-ketoisocaproate $(\alpha-\mathrm{KIC})$ as the substrate as described (13). The assay reaction mixture consisted of $1 \mathrm{mM} \alpha$-KIC, $30 \mathrm{mM}$ potassium phosphate, $2 \mathrm{mM} \mathrm{MgCl}_{2}, 0.4 \mathrm{mM}$ thiamine pyrophosphate, $0.4 \mathrm{mM}$ CoA, $1 \mathrm{mM} \mathrm{NAD}{ }^{+}, 0.1 \%$ (w/v) Triton X-100, $2 \mathrm{mM}$ DTT, pig heart dihydrolipoamide dehydrogenase ( 5 units $/ \mathrm{ml})$, and $2 \mu$ l of tissue extract. Absorbance at $340 \mathrm{~nm}$ was recorded. One unit of BCKD enzyme activity was defined as the formation of $1 \mu \mathrm{mol}$ of $\mathrm{NADH}$ per minute at $30^{\circ} \mathrm{C}$.

For determination of branched-chain aminotransferase (BCAT) activity, frozen tissue (50-100 mg) was pulverized with a mortar and pestle cooled in liquid nitrogen. The powder was transferred to a cooled centrifuge tube and suspended in 10 volumes of ice-cold homogenization buffer consisting of $20 \mathrm{mM}$ EDTA, 20 mM EGTA, 0.4\% (w/v) 3-[(3-cholamidopropyl)dimethylammonio]-2-hydroxy-1-propanesulfonate, 5 mM DTT, $10 \mu \mathrm{l} / \mathrm{ml}$ protease inhibitor cocktail, and $25 \mathrm{mM}$ HEPES ( $\mathrm{pH}$ 7.4). The tissue homogenates were then subjected to three rounds of freeze-thaw sonication before centrifugation at $15,000 \mathrm{~g}$ for 30 minutes at $4^{\circ} \mathrm{C}$. The supernatant was used as a source for total BCAT activity. The BCAT-1 (BCAT in cytoplasm) and BCAT-2 (BCAT in mitochondria) isozyme activities were also measured individually after separating mitochondrial and cytosolic fractions using a mitochondria isolation kit, MITO-ISO1 (Sigma-Aldrich, St. Louis, Missouri, USA). All BCAT enzyme activities were measured by a continuous 96-well plate spectrophotometric assay method (14) using MultiSkan Spectrum (Thermo Labsystems, Stockholm, Sweden). One unit of BCAT enzyme activity was defined as $1 \mu \mathrm{mol} \mathrm{NADH}$ reduced per minute at $37^{\circ} \mathrm{C}$.

Western blot analysis. Anti-BCAT-2 antibody and recombinant human BCAT-2 were a generous gift of Susan Hutson of Wake Forest University (WinstonSalem, North Carolina, USA). The antibody was a polyclonal antibody raised in rabbit against purified human recombinant BCAT-2. Tissue samples were subjected to SDS/PAGE using a NuPAGE Novex 4-12\% Bis-Tris Gel (Invitrogen, Carlsbad, California, USA). Ten micrograms of total protein were loaded onto each lane. Recombinant human BCAT-2 $(0.7 \mu \mathrm{g})$ was included as a standard. Resolved proteins were transferred to Immobilon P membranes. Membranes were blocked with $1 \%$ BSA/PBS and incubated with rabbit anti-human BCAT-2 $(0.45 \mu \mathrm{g} / \mathrm{ml})$ antixbodies. Bound antibodies were detected with anti-rabbit IgG conjugated with alkaline phosphatase (Promega, Madison, Wisconsin, USA). The immunoreactive protein bands were visualized using the bromochloroindolyl phosphate/nitro blue tetrazolium alkaline phosphatase substrate system according to the manufacturer's instruction (Sigma-Aldrich). 
Table 1

Blood amino acid concentrations in ENU mice

\begin{tabular}{lcccc}
\hline & \multicolumn{4}{c}{ Amino acid concentration $(\mu \mathrm{M})$} \\
Mouse & Valine & Leucine/Isoleucine & Arginine & Glycine \\
ENU control & $109 \pm 20.4^{\mathrm{A}}$ & $103 \pm 18.2^{\mathrm{A}}$ & $79 \pm 19.2^{\mathrm{A}}$ & $182 \pm 30.2^{\mathrm{A}}$ \\
$\begin{array}{l}n=328) \\
\text { Affected 1 }\end{array}$ & 2406.4 & 2336.3 & 305.6 & 231.7 \\
& $2885.3^{\mathrm{B}}$ & $2476.8^{\mathrm{B}}$ & $285.1^{\mathrm{B}}$ & $1930.6^{\mathrm{B}}$ \\
Affected 2 & 2494.3 & 2526.2 & 312.2 & 198.2 \\
Affected 3 & 3236.9 & 2720.9 & 266.7 & 268.6 \\
Affected 4 & 2427.7 & 2283.5 & 365.4 & 980.7 \\
\hline
\end{tabular}

${ }^{A}$ Control values are mean \pm SD. ${ }^{B}$ Second sample obtained 2 weeks later.

PCR and DNA sequencing. Genomic DNA was purified from $300 \mu \mathrm{l}$ whole blood using the Puregene DNA purification kit (Gentra Systems, Minneapolis, Minnesota, USA). All exons of candidate genes (Bcat1, Bcat2, and genes of the BCKD complex, including Bckdha, Bck$d h b, D b t$, and $D l d)$ were amplified and sequenced. Primers were designed by the Primer3 PCR primerpicking program (http://www-genome.wi.mit.edu/cgibin/primer/primer3_www.cgi). The primers used to detect exon 2 mutations in the Bcat2 gene were: $5^{\prime}$ GGGCTGGAGCTGACTTTAGTT, sense strand in intron 1 and 5'-CGCCAACCTGAGAGACTACA, anti-sense strand in intron 2. The PCR reactions were performed in a final volume of $50 \mu \mathrm{l}$, containing $0.2 \mu \mathrm{M}$ each primer, $10 \mathrm{mM}$ Tris- $\mathrm{HCl}$ ( $\mathrm{pH} 8.3$ ), $50 \mathrm{mM} \mathrm{KCl,1.5} \mathrm{mM} \mathrm{MgCl}_{2}$, $0.2 \mathrm{mM}$ deoxyribonucleoside triphosphate (dNTP), and 1 unit TaKaRa Taq (Takara, Shiga, Japan). Amplification conditions consisted of an initial denaturation of $3 \mathrm{~min}$ at $94^{\circ} \mathrm{C}$, followed by 20 cycles of touchdown PCR in 30 seconds at $94^{\circ} \mathrm{C}, 30$ seconds at $65^{\circ} \mathrm{C}$ (decrease $0.5^{\circ} \mathrm{C}$ per cycle), 40 seconds at $72^{\circ} \mathrm{C}$, and a final 20 cycles in 30 seconds at $94^{\circ} \mathrm{C}, 30$ seconds at $55^{\circ} \mathrm{C}$, and 40 seconds at $72^{\circ} \mathrm{C}$.

Total RNA was extracted from tissues using REzol C\&T (PROtech Technologies, Taiwan). First-strand cDNA was synthesized using oligo-dT15 primer and Moloney murine leukemia virus reverse transcriptase RNase $\mathrm{H}$ minus (Promega) in $25 \mu \mathrm{l}$ of $0.5 \mathrm{mM}$ dNTPs, $10 \mathrm{mM}$ Tris- $\mathrm{HCl}$ (pH 8.3), $2.5 \mathrm{mM} \mathrm{KCl}, 0.6 \mathrm{mM} \mathrm{MgCl}_{2}$, $20 \mathrm{U}$ RNase inhibitor, and $2 \mathrm{mM}$ DTT. RT-PCR amplification of $B$ cat 2 was conducted by using the following primer set: 5 '-CACACACCGGATCATGGCTG, sense strand in Bcat2 exon 1 and 5 '-CGCCTAGCAGAACGTAGCAT, antisense strand in Bcat2 exon4.

All amplified PCR fragments were purified using NucleoFast 96 PCR plate (Macherey-Nagel, Easton, Pennsylvania, USA), according to the manufacturer's instructions, to remove unincorporated primers and dNTPs. The purified PCR products were sequenced using the BigDye Terminator Cycle Sequencing Kit v1.1/3.1 (Applied Biosystems, Foster City, California, USA) following the manufacturer's instructions. Sequencing products were separated on either an ABI PRISM 3100 Genetic Analyzer or an ABI PRISM 3700 DNA Analyzer (Applied Biosystems). Raw sequencing data was analyzed by the DNA Sequencing Analysis Software v3.7 (Applied Biosystems).

Real-time quantitative RT-PCR. Real-time quantitative RT-PCR analysis was performed using the ABI PRISM 7700 Sequence Detection System (Applied Biosystems). PCR primers and TaqMan MGB probe for 18S RNA quantitation were purchased as an Assay-on-Demand kit from Applied Biosystems. The TaqMan MGB probe was linked to the reporter dye (6-FAM) at the $5^{\prime}$ end and to the nonfluorescent quencher (NFQ) at the $3^{\prime}$ end. For Bcat-2 mRNA quantitation, the primers and probes were designed using PrimerExpress (Applied Biosystems). EX-1,3 was designed to amplify specifically the alternative splicing transcript that has exon 2 deleted. EX-3,4 was designed to amplify the total Bcat-2 transcripts. EX-1,3 primers were: $5^{\prime}$-primer, $5^{\prime}$-CGCACACACCGGATCATG-3'; 3'-primer, 5'-CTTCTGTGGTTCTTTGGTCATCTGA-3'; and the probe was $5^{\prime}$-FAM-TCTGCAGCCTGTCCTAGTG-NFQ-MGB-3'. EX-3,4 primers were: $5^{\prime}$-primer, $5^{\prime}$-CCTGCTCTGGTCTGCACT-3'; $3^{\prime}$-primer, $5^{\prime}$-GTCTCCACCTTTGTATGCTTTCAAG-3'; and the probe was 5'-FAM-ACTCTCTGCAGCTCTTTG -NFQ-MGB-3'.

The cDNA corresponding to 75 ng of reversed transcribed total RNA was amplified in a final volume of $20 \mu \mathrm{l}$ using TaqMan Universal PCR Mastermix (Applied Biosystems) in duplicate assays for Bcat-2 Ex-1,3, Bcat-2 EX-3,4, and the endogenous 18S RNA. Final concentrations of PCR primers and TaqMan MGB probes were $900 \mathrm{nM}$ and $250 \mathrm{nM}$, respectively.

An analysis of the results was based on the Ct calculation, where $C t$ represents the cycle number at which fluorescence of the PCR samples crossed a given threshold. The expression level of the 18S RNA was taken as the first "calibrator" to normalize the total Bcat-2 mRNA in each tissue $(\Delta C t)$. The expression of the Bcat-2 in each of the control mouse tissue was then taken as the second "calibrator" to normalize the expression of Bcat-2 in the affected tissue accordingly $(\Delta \Delta C t)$. Final results were given as the relative amounts of Bcat- 2 mRNA in the affected mouse tissues as compared to the control.

\section{Results}

Mutant mice with an abnormal amino acid pattern. In screening 614 G3 mice from 39 families, we identified one mutant mouse having striking elevation of blood BCAA

\section{Table 2}

Real-time quantitative RT-PCR of Bcat-2 mRNA in mouse tissues

\begin{tabular}{lcccc} 
& \multicolumn{2}{c}{ Muscle } & \multicolumn{2}{c}{ Liver } \\
mRNA & Control & Affected & Control & Affected \\
$C t^{A}(B c a t-2)$ & 27.24 & 30.08 & 26.11 & 30.96 \\
$C t^{A}(18 S)$ & 19.56 & 17.26 & 16.46 & 17.53 \\
$\Delta C t^{B}$ & 7.68 & 12.82 & 9.65 & 13.43 \\
$\Delta \Delta C t^{C}$ & 0.00 & 5.14 & 0.00 & 3.79 \\
Relative expression level $^{\mathrm{D}}$ & $100 \%$ & $2.83 \%$ & $100 \%$ & $7.23 \%$
\end{tabular}

${ }^{A} C t$, cycle threshold. Values represent average of two determinations with variations $<3 \% .{ }^{B} \Delta C t=C t(B$ cat-2) $-C t(18 S)$. 18S RNA was used as an internal control. ${ }^{C} \Delta \Delta C t=\Delta C t$ (affected) $-\Delta C t$ (control). ${ }^{D}$ Expression level of $B c a t-2$ in affected mouse tissues relative to control, derived from $1 / 2^{\Delta \Delta C t}$. 
(valine, leucine/isoleucine, 20-30 fold) and moderate elevation of arginine (4-fold) (Table 1). Leucine and isoleucine were grouped together because they have the same molecular mass and are not differentiated by the tandem mass spectrometry method used in our laboratory. Using ion exchange chromatography (Beckman amino acid analyzer), the elevations of valine, leucine, and isoleucine were confirmed. In addition, it was found that leucine and isoleucine were present in a 60:40 ratio. The abnormal amino acid profile was confirmed in the second sample of the same mouse obtained 2 weeks later. Subsequently, we identified three more mutant mice in the same family having valine, leucine/isoleucine, and arginine elevations (Table 1). The finding of the same abnormal amino acid profile in multiply affected mice in independent litters of the same family suggested that this phenotype was heritable. In addition to the elevation of valine, leucine/isoleucine, and arginine, glycine was also elevated in two of the four mutant mice, although its elevation was not invariably seen even in the same mouse (Table 1). All mice had normal acylcarnitine profiles.

Other clinical phenotypes. In addition to the abnormal amino acid profile, these mutant mice (three males, one female) displayed failure to thrive (body weight $30-40 \%$ less than the unaffected siblings), weakness with decreased spontaneous movement, and hairs that were thin and scanty and with decreased luster. Family history revealed that many of the mutant mice expired prior to weaning and prior to metabolic screening at 2 months of age.

Why are these amino acids elevated? Since the most striking amino acid elevations were in valine, leucine, and isoleucine, all of which are BCAAs that share common metabolic pathways, we focused our investigations on these analytes. Conditions with elevated blood levels of BCAAs include starvation, diabetes mellitus, obesity, and inborn errors of metabolism (15). Starvation, diabetes, and obesity were ruled out in our mice; therefore, we considered the likelihood that the fundamental defect was an inborn error of BCAA catabolism.

$B C A A$ catabolism. BCAA oxidation begins with the transport of these amino acids into cells, where they undergo transamination, oxidative decarboxylation, and dehydrogenation (Figure 1). Transamination is catalyzed by BCATs that are either cytosolic or mitochondrial. Oxidative decarboxylation and dehydrogenase are catalyzed by a single BCKD complex, which is comprised of three catalytic components and two regulatory units (15).

Mammalian amino acid transporters have been classified into a distinct "system," depending upon substrate specificity, transport mechanism, and regulatory properties (16) There are more than 20 different transport systems known, which can fall into five superfamilies: two are preferentially $\mathrm{Na}^{+}$-coupled transporters and the other three often function as $\mathrm{H}^{+}$-coupled systems. BCAAs, along with other neutral amino acids, such as phenylalanine, tyrosine, and tryptophan, enter the cells through the sodium independent "L" system transporter (17). However, the unique amino acid pattern in our mutant mice did not support a defect in either the L system transporter or any of the known amino acid transporters.

To further investigate the abnormalities in the BCAA catabolism, we examined urine organic acids. Branchedchain $\alpha$-keto acids (BCKAs), 2-oxoisovaleric acid, 2-oxoisocaproic acid, and 2-oxo-3-methylvaleric acid were present in the affected mice but not in the normal mice (data not shown); these keto acids are transamination products of valine, leucine, and isoleucine, respectively. The elevation of BCAAs as well the corresponding BCKAs suggest that the block may be at the BCKD complex (Figure 1): the enzyme complex catalyzes the decarboxylation and dehydrogenation steps of BCKAs, and, when deficient, causes human MSUD. Clinically, MSUD patients present in early infancy with failure to thrive, poor feeding, lethargy, ketoacidosis, and, if left untreated, early death (15). These phenotypic features are similar to what we observed in our mice. The BCKD complex activity in both liver and muscle of our mutant mice, however, were normal (Table 1). BCKD comprises three catalytic components: a thiamine pyrophosphate-dependent decarboxylase (E1 $\alpha$, E13), a transacyclase (E2), and a dehydrogenase (E3). Sequencing the genes encoding these components revealed no mutation. Furthermore, no alloisoleucine, a pathognomonic marker for human MSUD (18), was detected in the urine of the affected mice.

Defect in BCAT. The possibility that a defect in the BCAT is responsible for the MSUD-like mice was investigated. In mammals, there are two BCAT isozymes, a cytosolic (BCATc or BCAT-1) and a mitochondrial (BCATm or BCAT-2) form (19) (Figure 1);

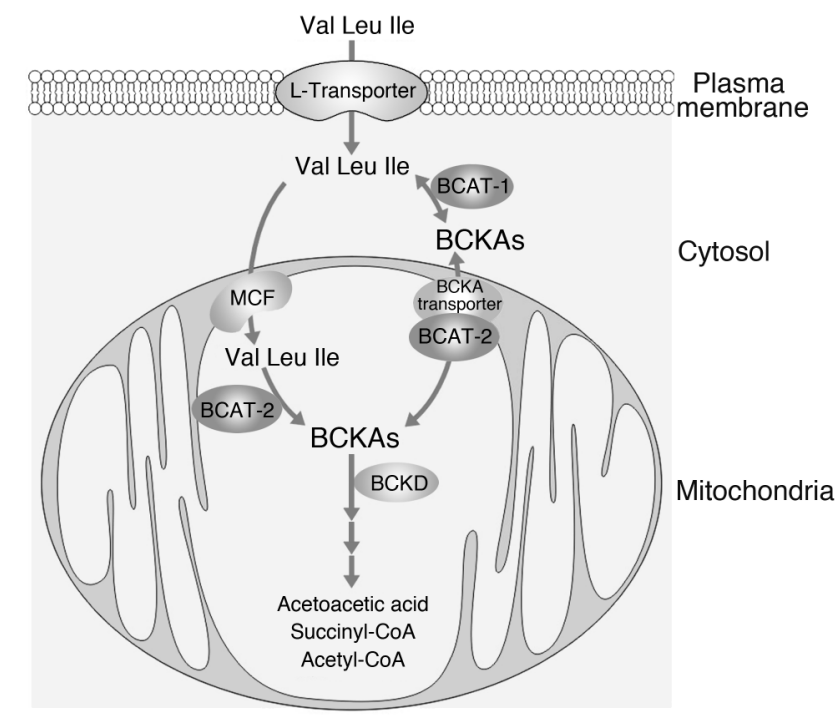

Figure 1

Schematic catabolic pathway of branched-chain amino acids valine (Val), leucine (Leu), and isoleucine (Ile). MCF, mitochondrial carrier family. 
a
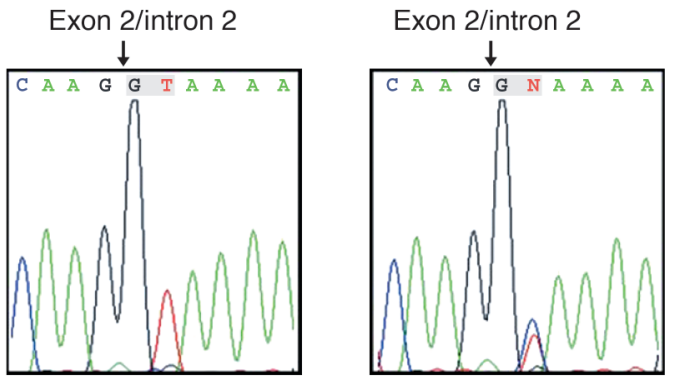

b

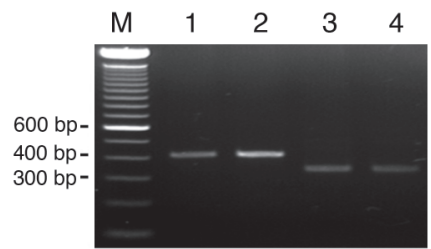

C

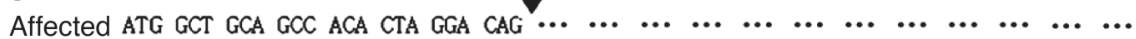
III III III III III III II| III

WT ATG GCT GCA GCC ACA CTA GGA CAG GTC TGG GCC OGA AAG CTT CTG CCT GTT CCC TGG CTT $\begin{array}{llllllllllllllllllll}M & A & A & A & T & L & G & Q & \mathbf{V} & \mathbf{F} & \mathbf{A} & \mathbf{R} & \mathbf{K} & \mathbf{L} & \mathbf{L} & \mathbf{P} & \mathbf{V} & \mathbf{P} & \mathbf{W} & \mathbf{L} \\ \end{array}$ $\downarrow$

GCT GCA GAC CTC CAG ATT CAG III 111 III 111 III 111 III CTA TGT GGG TCC AAA AGA TGT GTG TCC TCC ATA TTC AAG GCT GCA GAC CTC CAG ATT CAG

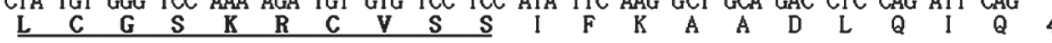

ATG ACC AAA GAA CCA CAG AAG AAG CCA GCC CCT AGC CAG GCA CTG CTG TTT GGG AAG ACC

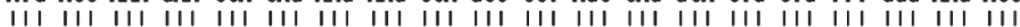
ATG ACC AAA GAA CCA CAG AAG AAG CCA GCC CCT AGC CAG GCA CTG CTG TTT GGG AAG ACC $\begin{array}{lllllllllllllllllll}M & T & K & E & P & Q & K & K & P & A & P & S & Q & A & L & L & F & G & K\end{array}$

TTC ACA GAC CAC ATG CTG ATG GTG GAG TGG AAT AAC AAG GCT GGA TGG GGC CCA CCA AGG

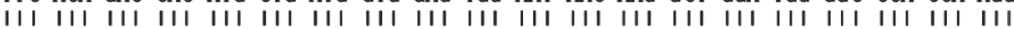
TTC ACA GAC CAC ATG CTG ATG GTG GAG TGG AAT AAC AAG GCT GGA TGG GGC CCA CCA AGG F T D H M L H V E W N N N A G W G P

ATC CAG CCC TTC CAG AAC CTC ACG CTA CAC CCA GCC TGC TCT GGT CTG CAC TAC TCT CTG

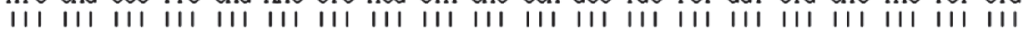
ATC CAG CCC TTC CAG AAC CTC ACG CTA CAC CCA GCC TGC TCT GGT CTG CAC TAC TCT CTG

$\begin{array}{lllllllllllllllllllll}\mathrm{I} & \mathrm{Q} & \mathrm{P} & \mathrm{F} & \mathrm{Q} & \mathrm{N} & \mathrm{L} & \mathrm{T} & \mathrm{L} & \mathrm{H} & \mathrm{P} & \mathrm{A} & \mathrm{C} & \mathrm{S} & \mathrm{G} & \mathrm{L} & \mathrm{H} & \mathrm{Y} & \mathrm{S} & \mathrm{L} & 100\end{array}$

\section{Figure 2}

Sequence analysis of mouse Bcat2 gene. (a) Nucleotide sequences showing $\mathrm{T} / \mathrm{T}$ (WT, left panel), $\mathrm{T} / \mathrm{C}$ (G1 father, middle panel) and C/C (affected, right panel) at second nucleotide position of intron 2. (b) RT-PCR results of mRNA amplified with primers located in exon 1 and exon 4 as described in Methods. Lane 1, WT liver; lane 2, WT muscle; lane 3 affected liver; lane 4, affected muscle; $M$, molecular weight markers. (c) Alignment of nucleotide and deduced amino acid sequences derived from RT-PCR results in $\mathbf{b}$. Mitochondrial targeting leader sequences are underlined. Dots represent gaps. Sequences marked between two arrows represent exon 2 . each is encoded by a single gene. Interestingly, BCAT-2 may also play a role in the transport of BCKAs, either it functions as a transport protein that transports BCKAs across the mitochondrial membrane, or it interacts with the inner mitochondrial membrane for BCKAs transport $(20,21)$.

Sequencing the Bcat genes revealed no mutation in the Bcat1 gene. However, a homozygous $\mathrm{T}$ to $\mathrm{C}$ nucleotide change in the $5^{\prime}$ splicing site consensus sequence of the exon 2 and intron 2 (IVS2 $+2 \mathrm{~T}>\mathrm{C}$ ) of the Bcat 2 gene was identified (Figure $2 \mathrm{a}$ ). Both parents were heterozygous for the mutation. RT-PCR amplification of liver and muscle Bcat 2 mRNA with primers located in exon 1 and exon 4 resulted in DNA fragments of 414 bp in WT and 339 bp fragments in the affected mouse tissues, respectively (Figure 2b). Sequencing the RT-PCR products of the affected mouse tissues revealed that the entire exon 2 of Bcat 2 mRNA was deleted (Figure 2c). Real-time quantitative RT-PCR showed that the amounts of Bcat- 2 mRNA were markedly reduced in the affected mouse tissues $(2.83 \%$ in muscle and $7.23 \%$ in liver) as compared with the control (Table 2). The Bcat-2 mRNA detected in the affected mouse tissues was predominantly the spliced variant (exon 2 deleted), and this spliced variant was not detected in the control mouse tissues (data not shown), which was consistent with data obtained from the qualitative RT-PCR experiments (Figure 2b). These data indicated that the splicing site mutation resulted in a mutant form of Bcat-2 mRNA (lacking exon 2), which had a decreased stability.

Western blot analysis using anti-human BCAT-2 antibody detected mouse BCAT-2 protein in the WT mouse heart, kidney, and muscle but not in the affected mouse tissues (Figure 3). Enzyme activity measure-

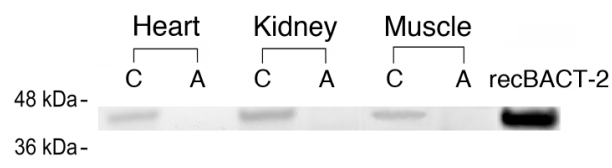

Figure 3

Western blot analysis of BCAT-2 in control (C) and affected (A) mouse tissues. Each lane contains $10 \mu \mathrm{g}$ of total protein from tissue extracts. Recombinant human BCAT-2 (recBCAT-2; $0.7 \mu \mathrm{g}$ ) was used as a standard. 
Table 3

BCKD complex and BCATs enzyme activities in mouse tissues

\begin{tabular}{|c|c|c|c|c|c|c|c|c|}
\hline \multirow[b]{3}{*}{ Tissue } & \multirow{2}{*}{\multicolumn{2}{|c|}{$\begin{array}{c}\mathrm{BCKD}(\mathrm{nmol} / \mathrm{min} / \mathrm{mg} \text { protein }) \\
\text { Actual }^{\mathrm{A}}\end{array}$}} & \multicolumn{6}{|c|}{ BCATs (nmol/min/mg protein) } \\
\hline & & & \multicolumn{2}{|c|}{ Total } & \multicolumn{2}{|c|}{ Cytosol } & \multicolumn{2}{|c|}{ Mitochondria } \\
\hline & Control & Affected & Control & Affected & Control & Affected & Control & Affected \\
\hline Muscle & 486.8 & 501.2 & 30.4 & 15.1 & 28.4 & 25.1 & 43.8 & $<0.1$ \\
\hline Liver & 349.2 & 333.6 & 22.3 & 29.4 & 22.4 & 35.1 & 17 & $<0.1$ \\
\hline
\end{tabular}

Values represent average of three determinations with variations $<5 \%$. ${ }^{A}$ Actual activity refers to $B C K D$ activity without dephosphorylation.

ments showed that total BCAT activity was decreased $50 \%$ in the affected mouse muscle (Table 3 ). However, when BCAT- 1 and BCAT-2 were measured separately, only mitochondrial BCAT activities were markedly reduced in the affected mouse tissues, whereas cytosolic BCAT activities were retained. These data established that the fundamental defect in our MSUD-like mice lies in the Bcat 2 gene. The fact that BCAT- 2 has a role in BCKA transport $(20,21)$ may explain why BCKAs (presumably derived from intact BCAT-1 activity) were elevated in our mice.

The spliced site mutation identified in our mutant mice caused a deletion of entire exon 2 of the Bcat- 2 mRNA. Because exon 2 contains the mitochondrial targeting leader sequence (Figure 2c), the deletion might be expected to have an effect on the BCAT- 2 targeting, as in the case of an alternatively spliced Bcat 2 variant mRNA detected in human tissues (22). This human spliced variant lacks 276 nucleotides in the $5^{\prime}$ end, including the entire mitochondrial targeting sequence. The spliced variant maintains the same reading frame and produces a truncated protein at a very low level in the cytosol (22). Whether the human spliced variant also has a decreased stability is not known. The pathogenesis of the mutation in our mutant mice, however, was primarily an unstable, mutant form of Bcat-2 mRNA (lacking exon 2) that resulted in the loss of BCAT- 2 protein and enzyme activity.

Response to a diet low in BCAAs. Our BCAT-deficient mice exhibited failure to thrive, weakness with decreased spontaneous movement, hair loss, and decreased luster. Without treatment, they died before 4 months of age. In an attempt to rescue these mice, we placed them on a low-BCAA diet by mixing human MSUD diet with regular rodent diet at a proportion of $2: 1$ or $3: 1$ (Figure 4). Human MSUD diet is an infant formula completely devoid of BCAAs that has been used successfully to treat human MSUD patients (23). Significant weight gains were seen on the low-BCAA diet (Figure 4a), along with improvements in hair quality and spontaneous activity. Concomitant with amelioration of clinical symptoms, BCAA levels decreased $(>85 \%)$ and arginine and glycine normalized (Figure 4b). Importantly, the mice became fertile and could be bred with the diet. A breeding colony had been established. All affected mice (now a total of 24) responded to the diet with an average of $44 \%$ weight gains and reversal of the clinical symptoms in 5 weeks.

\section{Discussion}

The BCAT-deficient mice had clinical and laboratory findings resembling human MSUD and, like human MSUD, responded to diet low in BCAAs. Compared with human patients, however, there were subtle differences, including elevations of arginine and glycine and higher levels of valine than leucine or isoleucine, whereas in the human, leucine is the highest among three elevated BCAAs. We hypothesize that arginine and glycine elevations are secondary to the increase in BCAAs, because BCAA restricted diet has resulted in normalization of these two amino acids in our mice. The difference between mouse and human with regard to individual BCAA elevations cannot be due to the diet, as both rodent and human diets have similar individual BCAA compositions. We reason that this difference is likely to
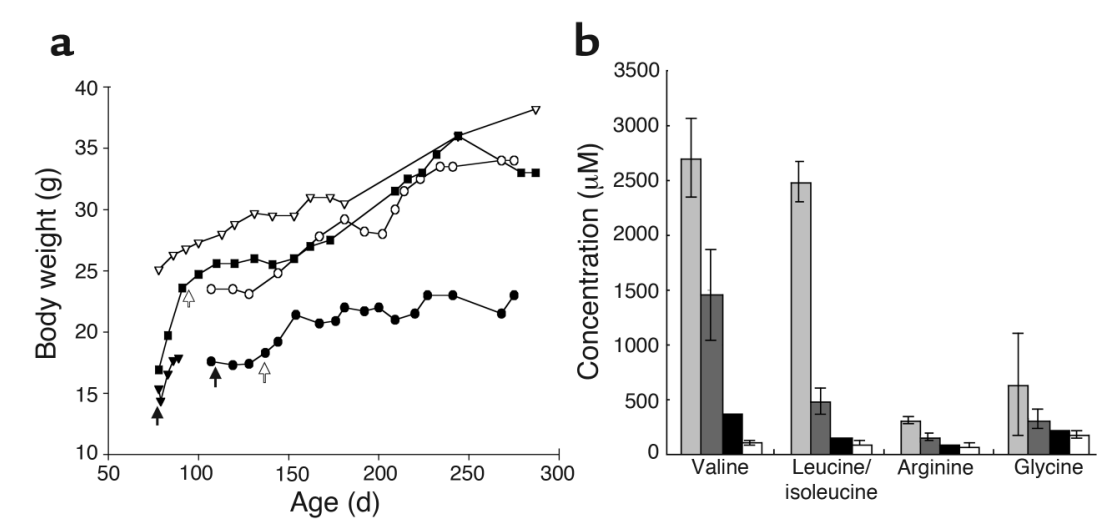

\section{Figure 4}

Body weight and amino acid changes with diet restricted in BCAAs. (a) Body weight changes of five mice in two litters: affected males (filled squares and filled triangles); affected female (filled circles); unaffected siblings (male, open triangles; female, open circles). Black arrows indicate when the restricted diet (MSUD diet/regular diet, 2:1) was initiated. Open arrows denote that diet was further changed to MSUD diet/regular diet, 3:1. One mouse (filled triangle) died accidentally at day 89. (b) Changes in amino acid concentrations. Light gray bars, affected mice with regular diet; dark gray bars, MSUD diet/regular diet, 2:1; black bars, MSUD diet/regular diet, 3:1; white bars, control ENU mice with regular diet. 
result from the fundamental genetic defect, which is BCAT-2 deficiency in our mice. BCAT- 2 might have a higher affinity for valine than leucine or isoleucine. It would be interesting to know whether there exist any unclassified human MSUD patients that might be due to BCAT-2 deficiency. They might be expected to have elevated BCAAs, especially valine, with no allo-isoleucine. Several early reports of patients with hypervalinemia, vomiting, failure to thrive, mental retardation, hyperkinesias, and nystagmus (24-26) suggest such patients may well exist and further study will be needed to confirm the presence of human BCAT-2 deficiency.

ENU mutagenesis is a powerful way to produce and screen genetic variants for gene function studies in the whole organism. A potential problem is the presence of other mutations induced by ENU, which may confound the observed phenotypes. In our study, we applied a three-generation strategy to detect recessive mutations. We have further bred the affected mice with WT mice and carried out outcross breeding. All mice, now through the sixth generation, continued to show $100 \%$ phenotype and genotype correlations due to the $B$ cat2 gene mutation. Other mutations, if present, are likely to be diluted out and contribute little, if any, to the observed phenotypes.

In conclusion, we have identified a mouse model with a novel enzyme deficiency resembling human MSUD. These mice could provide an important animal model for study of BCAA metabolism and its toxicity. Our data further suggest that metabolomics-guided screening, coupled with ENU mutagenesis, is a powerful approach to uncover novel enzyme deficiencies and recognize important pathways for genetic metabolic disorders.

\section{Acknowledgments}

We thank Yi-Jung Lin, Cheng-Chih Huang, and the National Genotyping Center for technical support and Mouse Mutagenesis Core for providing ENU mice. We thank Susan Hutson for providing the anti-BCAT-2 antibody and helpful discussion on the project. This work was supported by the National Science $\&$ Technology Program for Genomic Medicine from the National Science Council, Taiwan, and the Genomics and Proteomics Program from the Academia Sinica, Taiwan.

1. Fields, S. 1997. The future is function. Nat. Genet. 15:325-327.

2. Millington, D.S., Kodo, N., Terada, N., Roe, D., and Chace, D.H. 1991 The analysis of diagnostic markers of genetic disorders in human blood and urine using tandem mass spectrometry with liquid secondary ion mass spectrometry. Int. J. Mass Spectrom. Ion Process. 111:211-228.

3. Millington, D.S., Norwood, D.L., Kodo, N., Roe, C.R., and Inoue, F. 1989. Application of fast atom bombardment with tandem mass spectrometry and liquid chromatography/mass spectrometry to the analysis of acylcarnitines in human urine, blood, and tissue. Anal. Biochem. 180:331-339.
4. Millington, D.S., Kodo, N., Norwood, D.L., and Roe, C.R. 1990. Tandem mass spectrometry: a new method for acylcarnitine profiling with potential for neonatal screening for inborn errors of metabolism. J. Inherit. Metab. Dis. 13:321-324.

5. Chace, D.H., et al. 1995. Rapid diagnosis of maple syrup urine disease in blood spots from newborns by tandem mass spectrometry. Clin. Chem. 41:62-68.

6. Van Hove, J.L., et al. 1993. Medium-chain acyl-CoA dehydrogenase (MCAD) deficiency: diagnosis by acylcarnitine analysis in blood. Am. J. Hum. Genet. 52:958-966.

7. Rashed, M.S., Ozand, P.T., Bucknall, M.P., and Little, D. 1995. Diagnosis of inborn errors of metabolism from blood spots by acylcarnitines and amino acids profiling using automated electrospray tandem mass spectrometry. Pediatr. Res. 38:324--331.

8. Hrabe de Angelis, M.H., et al. 2000. Genome-wide, large-scale production of mutant mice by ENU mutagenesis. Nat. Genet. 25:444-447.

9. Nolan, P.M., et al. 2000. A systematic, genome-wide, phenotype-driven mutagenesis programme for gene function studies in the mouse. Nat. Genet. 25:440-443.

10. Rolinski, B., et al. 2000. The biochemical metabolite screen in the Munich ENU Mouse Mutagenesis Project: determination of amino acids and acylcarnitines by tandem mass spectrometry. Mamm. Genome. 11:547-551.

11. Weber, J.S., Salinger, A., and Justice, M.J. 2000. Optimal N-ethyl-Nnitrosourea (ENU) doses for inbred mouse strains. Genesis. 26:230-233.

12. Roe, C.R., et al. 1985. Diagnostic and therapeutic implications of medium-chain acylcarnitines in the medium-chain acyl-coA dehydrogenase deficiency. Pediatr. Res. 19:459-466.

13. Nakai, N., Kobayashi, R., Popov, K.M., Harris, R.A., and Shimomura, Y. 2000. Determination of branched-chain alpha-keto acid dehydrogenase activity state and branched-chain alpha-keto acid dehydrogenase kinase activity and protein in mammalian tissues. Methods Enzymol. 324:48-62.

14. Cooper, A.J., Conway, M., and Hutson, S.M. 2002. A continuous 96well plate spectrophotometric assay for branched-chain amino acid aminotransferases. Anal. Biochem. 308:100-105.

15. Chuang, D.T., and Shih, V.E. 2001. Maple syrup urine disease (branched-chain ketoaciduria). In The metabolic and molecular bases of inherited disease. C.R. Scriver, et al., editors. McGraw-Hill. New York, New York, USA. 1971-2005.

16. Hyde, R., Taylor, P.M., and Hundal, H.S. 2003. Amino acid transporters: roles in amino acid sensing and signalling in animal cells. Biochem. J. 373:1-18.

17. Le Cam, A., and Freychet, P. 1977. Neutral amino acid transport. Characterization of the A and L systems in isolated rat hepatocytes. J. Biol. Chem. 252:148-156.

18. Matthews, D.E., Ben-Galim, E., Haymond, M.W., and Bier, D.M. 1980. Alloisoleucine formation in maple syrup urine disease: isotopic evidence for the mechanism. Pediatr. Res. 14:854-857.

19. Davoodi, J., et al. 1998. Overexpression and characterization of the human mitochondrial and cytosolic branched-chain aminotransferases. J. Biol. Chem. 273:4982-4989.

20. Hutson, S.M., and Hall, T.R. 1993. Identification of the mitochondrial branched chain aminotransferase as a branched chain alpha-keto acid transport protein. J. Biol. Chem. 268:3084-3091.

21. Drown, P.M., Torres, N., Tovar, A.R., Davoodi, J., and Hutson, S.M. 2000. Use of sulfhydryl reagents to investigate branched chain alphaketo acid transport in mitochondria. Biochim. Biophys. Acta. 1468:273-284.

22. Than, N.G., Sumegi, B., Than, G.N., Bellyei, S., and Bohn, H. 2001. Molecular cloning and characterization of placental tissue protein 18 (PP18a)/human mitochondrial branched-chain aminotransferase (BCATm) and its novel alternatively spliced PP18b variant. Placenta. 22:235-243.

23. Snyderman, S.E., Goldstein, F., Sansaricq, C., and Norton, P.M. 1984 The relationship between the branched chain amino acids and their alpha-ketoacids in maple syrup urine disease. Pediatr. Res. 18:851-853.

24. Wada, Y., et al. 1963. Ideopathic hypervalinemia-probably new entity of inborn error of valine metabolism. Tohoku J. Exp. Med. 81:46-55.

25. Dancis, J., et al. 1967. Hypervalinemia. A defect in valine transamination. Pediatrics. 39:813-817.

26. Reddi, O.S., Reddy, S.V., and Reddy, K.R. 1977. A sibship with hypervalinemia. Hum. Genet. 39:139-142. 